\title{
PREDICTION OF HOURLY HEATING ENERGY USE FOR HVAC USING FEEDFORWARD NEURAL NETWORKS
}

\author{
Aleksandra Sretenović ${ }^{1}$ \\ Radiša Jovanović ${ }^{1}$, \\ Vojislav Novaković2 \\ Nataša Nord², \\ Branislav Živković ${ }^{1}$
}

\author{
${ }^{1}$ Faculty of Mechanical Engineering, \\ University of Belgrade, \\ Belgrade, Serbia \\ ${ }^{2}$ Department of Energy and Process \\ Engineering, Norwegian University of \\ Science and Technology, \\ Trondheim, Norway
}

\begin{abstract}
:
In this paper, feedforward neural network, one of the most widely used artificial intelligence methods, was proposed for the prediction of hourly heating energy use of one university campus. Two different approaches were presented: network that provides one output (heating energy use for selected hour) and network with 24 outputs (daily profile of heating energy use). The proposed models were trained and tested using real measured hourly energy use and meteorological data. It has been shown that both models can be used for the prediction with satisfying accuracy. This kind of prediction can be used for calculating accurate energy bills, which is very useful considering that the significant part of the campus is being leased. Estimating energy use for different weather conditions can help in energy planning.
\end{abstract}

\section{Keywords:}

neural networks, building energy use prediction.

\section{INTRODUCTION}

Building sector in Europe is responsible for $40 \%$ of total energy use and $36 \%$ of total $\mathrm{CO}_{2}$ emission [1], so in order to carry out adequate energy planning, precise building energy use prediction is necessary. Existing methods for building energy use calculation can be roughly divided into three categories: "white box", "gray box" and "black box" methods [2]. "White box" approach uses physical techniques to model building behavior. Various software solutions based on solving equations describing heat transfer can be found on the market. This kind of modeling requires extensive knowledge of various mechanisms occurring inside and outside of the building envelope, so in recent years, significant attention has been given to statistical methods that do not require any physical information. When using "black box" (statistical, data-driven) methods, it is required that all input and output variables are known and measured. Modeling consists of determination of a mathematical relationship between the independent and dependent variables. One of the most used methods is Artificial Neural Network. The review on the different neural network models used for building energy prediction can be found in [3]. Ekici and Aksoy [4] developed the backpropagation three-layered ANN for the prediction of the heating energy requirements of various buildings. 
In [5] the author used hourly heating energy use for a model house calculated by degree-hour method for training and testing the ANN model. Successful applications of ANN for prediction of building energy use can also be found in [6] and [7]. The main idea in this paper is to use real, measured energy use for training and testing the models.

\section{ARTIFICIAL NEURAL NETWORKS}

Artificial Neural Network (ANN) method is a computational intelligence technique, based on the information processing system of the human brain, which may be used as an alternative method in engineering analysis and prediction.

\section{Feedforward backpropagation neural network (FFNN)}

The feedforward neural network architecture consists of an input layer, an output layer, and one or more hidden layers of neurons. Each layer has a certain number of neurons which are fully interconnected with adaptable weighted connections to the neurons in the subsequent layer. The nonlinear activation functions in the hidden layer neurons enable the neural network to be a universal approximator. Training network comprises of adjustment of the weights, so that the network can produce the desired response to the given inputs. Various training algorithms could be applied to minimize the error function, but the most widely used is the backpropagation algorithm. It uses a gradient descent technique to minimize the cost function (mean square difference between the desired and the actual network outputs).

\section{CASE STUDY}

The Norwegian University of Science and Technology (NTNU) campus Gløshaugen is a typical representative of the group of "mixed use" buildings. It consists of $35 \mathrm{ob}-$ jects with significant energy use, such as classrooms, sport facilities, laboratories, kitchen, etc. The buildings are usually multi-functional, and most of them have laboratories, which indicate possible high energy use. The total campus area is approximately $300,000 \mathrm{~m}^{2}$. Building and Energy Management System (BEMS) and web-based Energy Monitoring System (Energy Remote Monitoring - ERM) are available for operation and energy monitoring at NTNU [8]. Hourly heat and electricity consumption from all installed meters and submeters can be collected on
ERM. The main heating use meter for the entire campus is located in Old Electric Building (Fig.1). It is installed by the district heating supplier, so these measurements are taken as relevant for creating the model.

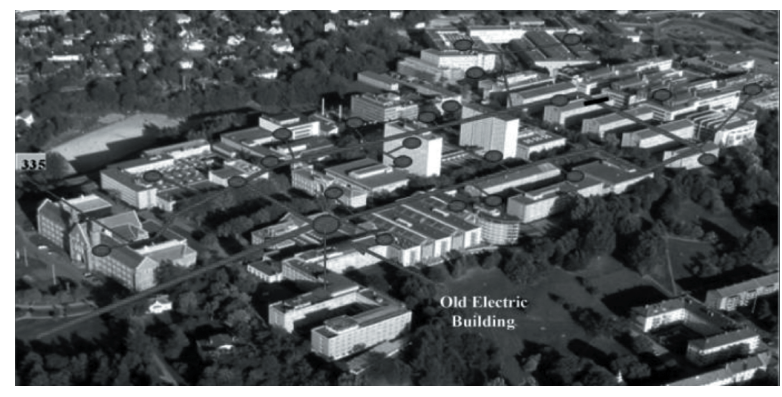

Fig. 1. District heating in NTNU campus Gløshaugen.

\section{MODEL DEVELOPMENT}

In previous work, various models for prediction of daily heating energy use and their improvements were proposed [9-12]. For training and testing the models, the coldest period for years 2009-2012 was selected. The proposed single models were: FFNN, Radial Basis Function Network (RBFN), Adaptive Neuro-Fuzzy System (ANFIS), Support Vector Machine (SVM). The innovative approach comprised of: using ensemble technique (combining outputs of individual networks in single prediction), multi-stage ensembles (using neural network for combining the outputs of individual models), using $\mathrm{k}$-means clustering for selecting ensemble members, $\mathrm{k}$ means clustering of input dataset. More details about these methodologies and achieved results can be found in [9-12]. The accuracy of the neural networks models for prediction of hourly heating energy use is investigated in this paper, as an extension of the previously published work.

For developing models and evaluating their prediction results, the coldest period for years 2009-2012 was used (the same as for modeling daily heating energy use). Data with obvious errors in measurement were removed from the dataset. In total, there were 309 days (7416 samples) for training period, and 98 days (2352 hourly heating consumption data) for testing period.

The selection of input variables has a critical role in building an ANN prediction model, and there is no general rule that can be followed in this process. In most cases, it depends on engineering judgement and experience and is carried out almost entirely by trial and error. However, some statistical analysis can be very helpful in 
determining variables with significant influence on the system output. Forward selection method was used to define the meteorological variables that have the biggest influence on hourly heating energy use. The results, as expected, showed that the mean hourly outside temperature is most highly correlated with the desired output variable.

For the prediction of hourly heating energy use two different models were developed. The first model (M1) is a FFN network with one output variable - hourly heating energy use, using following input variables: month of a year (M), day of a week (D), hour of a day (h), mean hourly outside temperature ( $\mathrm{t}$ ) and it is presented in Fig. 2.

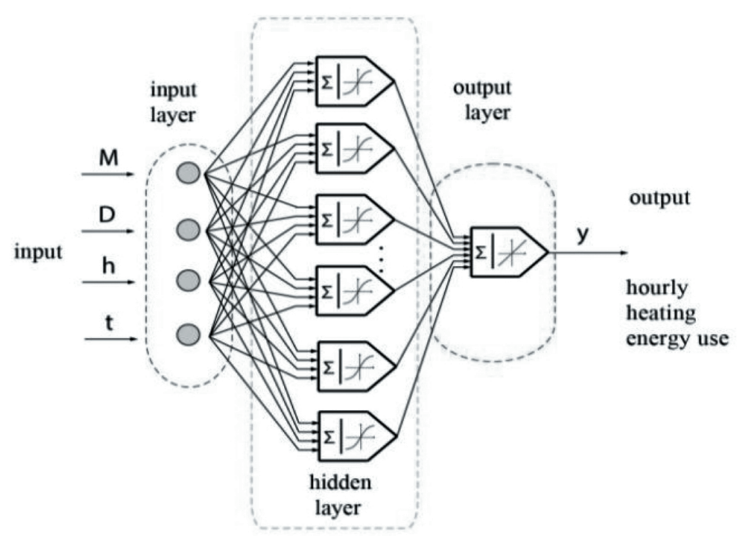

Fig. 2. FFNN model with 4 inputs and 1 output.

In order to examine the possibility of predicting the daily profile of heating energy use, the second model (M2) was considered. Model M2 is similar to M1, with the following modifications: instead of using the hour of the day and mean hourly outside temperature as input variables, the daily temperature profile was used as input ( 24 values of mean hourly outside temperatures). In this case, there are 24 output values which represent predicted hourly heating use. This model, which gives the daily profile of district heating use in campus, is presented in Fig. 3.

It should be noted that both proposed models are static prediction models: they do not use output history (previous heating energy use) as an additional input variable.

For both models, three layer feedforward neural networks layer composed of one input layer, one output layer and one hidden were used. The activation functions used for the hidden and output layers were the hyperbolic tangent and linear functions, respectively. For various numbers of neurons in hidden layers of both models 20 networks were trained according to the LevenbergMarquardt optimization algorithm, using different initial weights. All input and output variables were normalized to the interval $(0,1)$ before training to ensure that no special variable is dominant over the others. The number of hidden neurons was identified using a trial and error procedure and it was found to be 10 neurons for $\mathrm{M} 1$ and 12 neurons for M2.

To evaluate the obtained results, the coefficient of determination $\left(\mathrm{R}^{2}\right)$, root mean square error (RMSE) and mean absolute percentage error (MAPE) were used as indices of the prediction accuracy for developed networks.

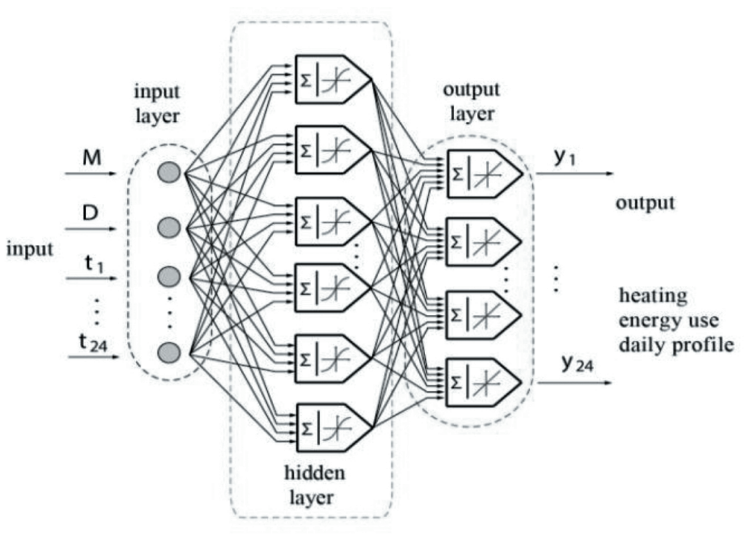

Fig. 3. FFNN model with 26 inputs and 24 outputs.

\section{RESULTS AND DISCUSSION}

Similarly to the previously mentioned work, the potential improvement of accuracy by adding previous heating energy use as additional input variable should be investigated. For the daily heating energy use of campus, prediction was more accurate when the energy use of the previous day was an input. But, it is necessary to have all input variables for the selected prediction period. That is the reason why these kinds of predictions are usually done one day ahead. The other possibility is to develop models to separately predict these input variables and then use them to predict energy use. These are challenges for application of almost all of different prediction methods in practice, especially for online types. In this paper, it is decided that the inputs are only the most influencing meteorological variables (outside air temperature).

Prediction indices are shown in Tab. 1. The results show that the network with 24 outputs achieves better prediction accuracy, both in training (MAPE $=7.72 \%$ ) and testing period (MAPE=10.93\%), comparing to FFNN with one output, with error of $9.53 \%$ and $11.26 \%$ for training and testing database, respectively. The comparison between the measured data for the selected days in test period and prediction of FFNN model with 1 output and 24 outputs are shown in Fig.4 and Fig. 5. 


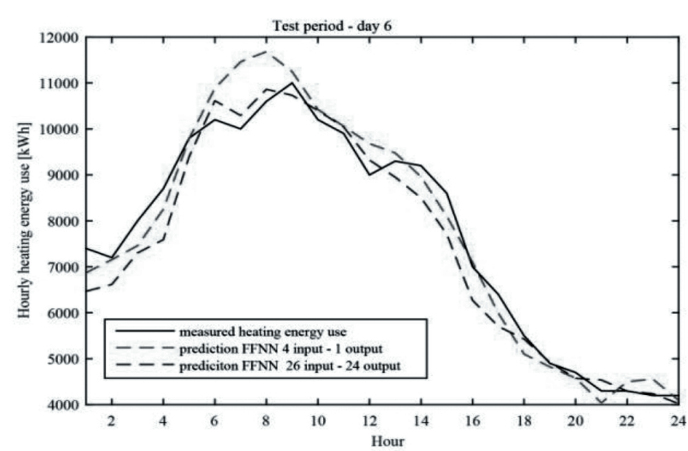

Fig. 4. Comparison of measured hourly heating use and prediction of the FFNN models for one day in test period (day 6).

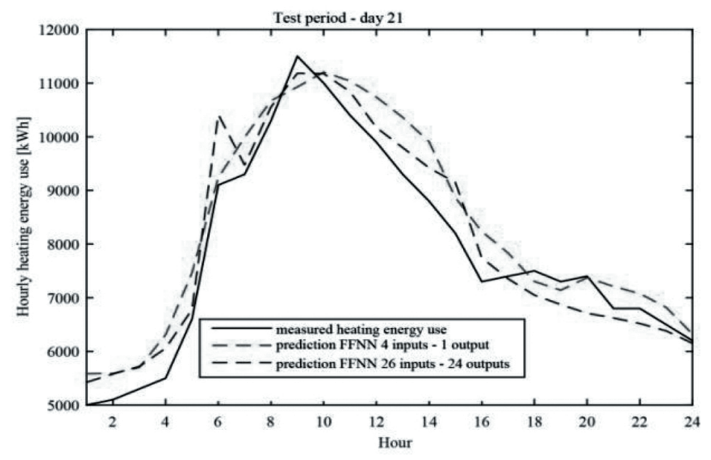

Fig. 5. Comparison of measured hourly heating use and prediction of the FFNN models for one day in test period (day 21).

\begin{tabular}{cccc}
\hline & & $\begin{array}{c}\text { FFNN } \\
\text { 4 inputs - } \\
\text { 1 output }\end{array}$ & $\begin{array}{c}\text { FFNN } \\
\text { 26 inputs - } \\
\text { 24 outputs }\end{array}$ \\
\hline $\begin{array}{c}\mathbf{R}^{2} \\
{[-]}\end{array}$ & Training & 0.9339 & 0.9602 \\
\cline { 2 - 4 } & Test & 0.9281 & 0.9255 \\
\hline $\begin{array}{c}\text { MAPE } \\
{[\%]}\end{array}$ & Training & 9.5298 & 7.7253 \\
\cline { 2 - 4 } & Test & 11.2615 & 10.9302 \\
\hline RMSE & Training & 757.79 & 605.92 \\
\cline { 2 - 4 }$[\mathrm{kWh}$ & Test & 758.39 & 746.06 \\
\hline
\end{tabular}

Table 1. Prediction quality indicies.

It can be seen that the output profiles of both models adequately match the measured district heating use. Fig. 6 and Fig. 7 show MAPE by hour for training and testing period, respectively. It can be seen that the biggest errors, both for training and testing period, occur in early morning working hours. MAPE for FFNN model with one output goes up to $20 \%$. These significant deviations have effect on total model prediction error and they indicate that the warm-up period of the campus should be taken into account. Slightly higher errors are also obvious in the period after working hours, when the heating energy use is not so correlated mainly with outside air temperature, but it also depends on the heating setpoint.

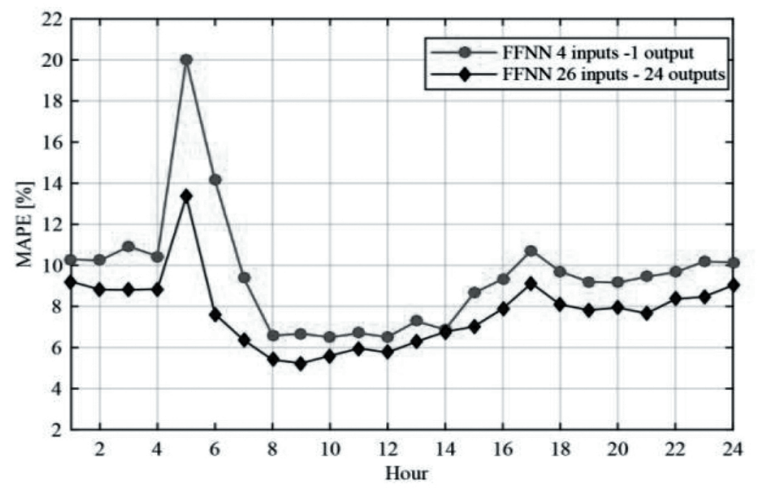

Fig. 6. MAPE for training perid by hours.

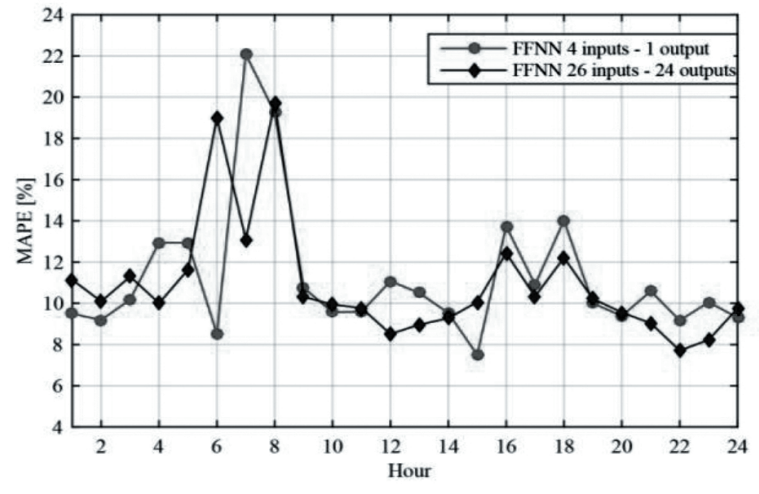

Fig. 7. MAPE for testing period by hours.

\section{CONCLUSION}

In this paper, prediction of hourly heating energy use for university campus Gløshaugen was investigated. For training models, the coldest period for 2009-2011 (7416 data samples) and for testing 2012 (2352 samples) were used. Two different FFNN models were developed: M1 FFNN with 4 inputs (M, D, t, h) and one output (hourly heating energy use) and M2 -FFNN with 26 inputs (M, D, daily temperature profile) and 24 outputs (daily heating energy use). It is shown that both models can be used for the prediction of hourly heating energy use, while better results are achieved with FFNN with 24 outputs. The error achieved with $\mathrm{M} 1$ for test period is $\mathrm{MAPE}=11.26$, and for M2 is $10.93 \%$. These models may be used to recover the lost data on heating energy use for the purpose of 
issuing correct energy bills, since the significant part of the campus is leased to other users. Also, if predicted values of previously successfully trained models are significantly different from energy use read on the meter, it can indicate meter malfunctioning. It is possible to extend the database, by analyzing more similar objects and taking into account additional input variables. This kind of prediction of energy use for different weather conditions can help in future energy planning. It is also expected that predictive models using artificial intelligence will be more widely used in industry in order to accurately estimate energy use of complex systems with significant number of variables.

\section{ACKNOWLEDGMENT}

Data used for this paper were gathered during study visit to NTNU, as a part of the collaborative project "Sustainable Energy and Environment in Western Balkans". The project was funded through the Norwegian Programme in Higher Education, Research and Development in Western Balkans, Programme 3: Energy Sector (HERD Energy) for the period 2011-2013.

\section{REFERENCES}

[1] C ouncil., E.P.a., Directive 2010/31/EU of the European Parliament and of the Council of 19 May 2010 on the energy performance of buildings. Official Journal of the European Union, 2010. L153: pp. 13-35.

[2] Zhao, H.-x. and F. Magoulès, "A review on the prediction of building energy consumption". Renewable and Sustainable Energy Reviews, vol. 16, 2012, pp. 3586-3592.

[3] Kumar, R., R. Aggarwal, and J. Sharma, "Energy analysis of a building using artificial neural network: A review", Energy and Buildings, vol. 65, 2013, pp. 352-358.
[4] Ekici, B.B. and U.T. Aksoy, "Prediction of building energy consumption by using artificial neural networks", Advances in Engineering Software, vol. 40, 2009, pp. 356-362.

[5] Dombayc1, Ö.A., "The prediction of heating energy consumption in a model house by using artificial neural networks in Denizli-Turkey", Advances in Engineering Software,vol. 41, 2010, pp. 141-147.

[6] González, P. A. and Zamarreno, J. M. "Prediction of hourly energy consumption in buildings based on a feedback artificial neural network". Energy and Buildings, vol. 37, 2005, pp. 595-601.

[7] Karatasou, S., Santamouris, M. and Geros, V. "Modeling and predicting building's energy use with artificial neural networks: Methods and results", Energy and Buildings, vol. 38, 2006, pp. 949-958.

[8] Guan, J., N. Nord, and S. Chen, "Energy planning of university campus building complex: Energy usage and coincidental analysis of individual buil dings with a case study", Energy and Buildings, vol. 124, 2016, pp. 99-111.

[9] R. Jovanović and A. Sretenović, "Various multistage ensembles for prediction of heating energy consumption", vol.36, 2015, pp.119-132.

[10] R. Jovanović, A. Sretenović and B. Živković, "Ensemble of various neural networks for prediction of heating energy consumption", Energy and Buildings, vol. 94, 2015, pp. 189-199.

[11] R. Jovanović, A. Sretenović, and B. Živković, "Multistage ensemble of feedforward neural networks for prediction of heating energy consumption", Thermal Science, vol. 20, 2016, pp. 1321-1331.

[12] R. Jovanović, and A. Sretenović, "Ensemble of radial basis neural networks with k-means clustering for heating energy consumption prediction", FME Transactions, vol. 45, 2017, pp. 51-57. 